\title{
Placebo responders and nonresponders: what's new?
}

\author{
Elisa Frisaldi*,1, Aziz Shaibani ${ }^{2}$ \& Fabrizio Benedetti ${ }^{1,3}$ \\ ${ }^{1}$ Department of Neuroscience, University of Turin Medical School, Corso Raffaello 30, 10125 Turin, Italy \\ ${ }^{2}$ Nerve \& Muscle Center of Texas, 6624 Fannin St Suite 1670, Houston, TX, USA \\ ${ }^{3}$ Plateau Rosà Laboratories, Plateau Rosà, Italy/Switzerland \\ *Author for correspondence: Tel.: +0039 011 6708491; elisa.frisaldi@unito.it
}
"This mechanism can clinically be exploited by providing precise a priori information concerning a treatment, which will help to create precise and specific expectations."

First draft submitted: 21 July 2018; Accepted for publication: 31 July 2018; Published online: 21 November 2018 Keywords clinical trials $\bullet$ computational neuroscience $\bullet$ expectations $\bullet$ learning $\bullet$ neuroimaging $\bullet$ personality $\bullet$
pharmacogenomics $\bullet$ placebo effect $\bullet$ placebo responders $\bullet$ treatment moderators

Over the last 5 years new insights into the neuroscience of the placebo effect and thus into the possibility to identify placebo responders or nonresponders have emerged [1], as emphasized by a group of internationally recognized placebo researchers [2]. These experts agree on the importance to differentiate between placebo and nocebo responses versus placebo and nocebo effects. Indeed, the placebo and nocebo response includes all health changes that result after administration of an inactive treatment, among which natural history and regression to the mean. Otherwise, the placebo and nocebo effect refers to the changes specifically attributable to placebo and nocebo mechanisms, including the neurobiological and psychological mechanisms of expectancies. Importantly, not only do placebos and nocebos have effects during the prescription of placebo treatments; they can also substantially modulate the efficacy and tolerability of the active treatment, be it pharmacological or not.

Since placebo effects are triggered by complex central nervous system mechanisms, such as expectations and learning processes, it is reasonable to assume that specific brain measures may identify placebo responders and nonresponders. From this perspective, the attempt to use neuroimaging technologies has recently produced new findings, particularly in the field of placebo analgesia. Prefrontal circuitry has always been found to be involved in placebo analgesia in studies on chronic back pain [3], fibromyalgia [4] and chronic knee osteoarthritis [5]. The right midfrontal gyrus connectivity was specifically identified as a biomarker that can predict the placebo response in patients affected by osteoarthritis [5]. Interestingly, activity in the right midfrontal gyrus was observed to be related to decision making, memory and planning processes, thus supporting the idea that placebo analgesia is driven by a complex top-down modulation. The amygdala, nucleus accumbens (NAc), and ventral striatum (VS) were also found to be regions closely connected to the medial prefrontal circuitry. Placebo analgesic treatments reliably reduce activity in the amygdala and increase activity in the NAc-VS region [6]. In particular, interindividual differences in NAc-VS seem to be important for identifying placebo responders and nonresponders. Indeed, strong placebo analgesic effects are predicted by NAc-VS activity including: stronger placebo-related opioid and fMRI activity responses during pain, increased grey matter volume and stronger fMRI responses in a reward pursuit task unrelated to pain [6].

Beside these advances in neuroimaging, the pharmacological experimental approach has shown that placebo analgesic effects can be boosted by using both vasopressin and oxytocin agonists, and that oxytocin modulation of placebo effects seems to be dose dependent $[7,8]$. More specifically, a randomized trial on healthy participants showed that intranasal vasopressin ( $40 \mathrm{IU})$ increases placebo analgesia significantly as compared to no treatment, intranasal oxytocin (24 IU) and intranasal saline $(0.4 \mathrm{ml})$, and these effects were found only in women. Conversely to what was found in subjects who inhaled a higher dose of oxytocin (40 IU) [8], a 24 IU of intranasal oxytocin did not enhance placebo effects in either sex. Moreover, women with both lower dispositional anxiety and cortisol levels 
showed the largest vasopressin-induced modulation of placebo effects, suggesting a moderating interplay between pre-existing psychological factors and cortisol changes [7].

Personality traits also contribute to explain a substantial proportion of the variance in placebo analgesic effects; placebo effects have been found to be related to optimism, suggestibility, empathy and neuroticism, whereas nocebo effects have been linked to pessimism, anxiety and catastrophizing [9]. Attempts to describe the placebo responder or nonresponder through a single personality trait may be limiting and, indeed, a transactional model of placebo responding, in which dispositional characteristics dynamically interact with environmental contingencies, was presented in 2015 [10]. According to this model, the overlaps among the personality traits identified so far suggest that placebo responsiveness could be conceptualized in terms of a two-faceted construct consisting of an inward and an outward orientation. Persons with 'inward orientation' show a tendency to have an internal focus, or the ability to respond to suggestions regarding internal experience, while persons with 'outward orientation' show perviousness to external inputs. Absorption, suggestibility and acquiescence are personality traits that can be included in the first facet of placebo responsiveness, whereas resiliency, altruism, straightforwardness, optimism, extraversion and dopamine related traits belong to the second facet of placebo responsiveness. When a match between the type of individual and the nature of the contextual cues is missing, then responding may not arise. Consequently, health practitioners could maximize the placebo component of any treatment or increase the chance of responding to treatment by tailoring clinical approaches based on how certain individuals respond to contextual treatment. In a recent experimental model of conditioning and heat thermal painful stimulation, the potential advantage of considering several personality factors was confirmed [9]. Moreover, the same authors showed, for the first time, that expectations predict both placebo and nocebo effects independently of personality factors. In fact, expectations were found to highly correlate to placebo and nocebo effects, whereas psychological factors per se did not influence level of expectations of either reduction or increase in pain.

Although the most recent insights into the mechanisms of placebo effect are related to placebo analgesia, important advances have also been made in Parkinson's disease [11,12], and in both psychiatric [13-15] and gastrointestinal disorders [16]. Furthermore, it is worth remembering that placebo researchers have learned that genes can also modify the placebo effect and, in light of the involvement of many neurotransmitters in pain and reward, this is not surprising. Recently, a homozygous state for methionine in the COMT Val158Met polymorphism predicted the higher placebo effect in patients suffering from irritable bowel syndrome [17]. Since this study included a no treatment control group, it was possible for the first time to demonstrate genetic modulation of a true placebo effect disentangled from changes related to regression to the mean, spontaneous recovery and natural fluctuations of illness. Beside the COMT gene, whose expression influences the brain's levels of the neurotransmitter dopamine, there is evidence for genetic variation in other neurotransmitter pathways. These include the opioid, endocannabinoid and serotonin pathways, theorizing the potential existence of a placebome or 'network' of genes [17]. Both drugs and placebos would act on these neurotransmitter pathways, which suggests that a drug could potentially change a placebo effect and, the other way round, a placebo effect can modify a drug effect. Knowledge of the placebome has the potential to guide development of novel strategies for both identifying placebo responders and improving clinical trials design. Needless to say, many attempts have been made to increase the efficiency of randomized clinical trials (RCTs) with placebo run-in periods that eliminate placebo responders. A priori elimination of high placebo responders in RCTs on the basis of a genetic screening could represent a new way of running a clinical trial, although this should be confirmed by further research and it would raise important ethical and clinical questions.

There is today a growing need to better understand the psychological and biological underpinnings of placebo effects, particularly in the setting of clinical trials, whereby many psychological factors can be assessed in more detail. For example, the Stanford Expectations of Treatment Scale [18], aimed at assessing pretreatment positive and negative expectations, has been found to predict between 12 and $18 \%$ of outcome variance in pain patients. Consistently with this, the impact of positive or negative expectations on the therapeutic outcome has recently been proven in RCTs in depression and Parkinson's disease [14,19]. The need to measure patients' expectation of benefit from baseline to the end of a clinical trial, and to identify those participants susceptible to a placebo effect, will be the challenge of the near future.

Last but not least, computational neuroscience has recently emerged as a tool to better understand information processing and brain functions involved in the placebo effect. For example, focusing on placebo analgesia from a Bayesian predictive-coding perspective was a starting point to formally develop a model, which adequately combines the precision and magnitude of prior expectations on placebo effects [20]. This model postulates that pretreatment expectations are integrated with incoming nociceptive information, and both are weighted by their 
relative precision to form the pain percept and placebo effect. In a clinical context, these studies clearly indicate that the level of precision of prior treatment experiences and associated expectations is a crucial determinant of placebo effects in treatment outcomes. This mechanism can clinically be exploited by providing precise $a$ priori information concerning a treatment, which will help to create precise and specific expectations. Beside computational neuroscience, the machine learning approach systematically examines how interactions between different moderators, both person specific and study specific, can better capture the richness of human complexity when pursuing to identify drug versus placebo responders [15].

Progress has thus been made over the past few years, and we have now more pieces of information that can be harnessed both in the clinical trial setting and in medical practice. However, further research needs to provide more details in order to integrate all these findings and thus enhancing the contribution and specificity of any single approach, from neurophysiology to psychology, neuroimaging, pharmacology, genomics, computational neuroscience and machine learning.

Financial \& competing interests disclosure

This work was supported by Innovative Clinical Trials and \& Healthcare (ICTH) Initiative (Italy/Switzerland) and Nerve \& Muscle Center, Houston, TX, USA. The authors have no other relevant affiliations or financial involvement with any organization or entity with a financial interest in or financial conflict with the subject matter or materials discussed in the manuscript apart from those disclosed.

No writing assistance was utilized in the production of this manuscript.

\section{References}

1 Benedetti F, Frisaldi E. Creating placebo responders and nonresponders in the laboratory: boons and banes. Pain Manag. 4(3), 165-167 (2014).

2 Evers AWM, Colloca L, Blease C et al. Implications of placebo and nocebo effects for clinical practice: expert consensus. Psychother. Psychosom. 12(4), 204-210 ( 2018).

3 Hashmi JA, Baria AT, Baliki MN, Huang L, Schnitzer TJ, Apkarian AV. Brain networks predicting placebo analgesia in a clinical trial for chronic back pain. Pain 153(12), 2393-2402 (2012).

4 Schmidt-Wilcke T, Ichesco E, Hampson JP et al. Resting state connectivity correlates with drug and placebo response in fibromyalgia patients. Neuroimage Clin. 6, 252-261 (2014).

5 Tétreault P, Mansour A, Vachon-Presseau E, Schnitzer TJ, Apkarian AV, Baliki MN. Brain connectivity predicts placebo response across chronic pain clinical trials. PLoS Biol. 14(10), e1002570 (2016).

6 Wager TD, Atlas LY. The neuroscience of placebo effects: connecting context, learning and health. Nat. Rev. Neurosci. 16(7), 403-418 (2015).

7 Colloca L, Pine DS, Ernst M, Miller FG, Grillon C. Vasopressin boosts placebo analgesic effects in women: a randomized trial. Biol. Psychiatry 79(10), 794-802 (2016).

8 Kessner S, Sprenger C, Wrobel N, Wiech K, Bingel U. Effect of oxytocin on placebo analgesia: a randomized study. JAMA 310(16), 1733-1725 (2013).

9 Corsi N, Colloca L. Placebo and nocebo effects: the advantage of measuring expectations and psychological factors. Front. Psychol. 8, 308 (2017).

10 Darragh M, Booth RJ, Consedine NS. Who responds to placebos? Considering the "placebo personality" via a transactional model. Psychol. Health Med. 20(3), 287-295 (2015).

11 Benedetti F, Frisaldi E, Carlino E et al. Teaching neurons to respond to placebos. J. Physiol. 594(19), 5647-5660 (2016).

12 Shin CW, Hahn S, Park BJ, Kim JM, Park EO, Jeon B. Predictors of the placebo response in clinical trials on Parkinson's disease: a meta-analysis. Parkinsonism Relat. Disord. 29, 83-89 (2016).

13 Weimer K, Colloca L, Enck P. Placebo effects in psychiatry: mediators and moderators. Lancet Psychiatry 2(3), 246-257 (2015).

14 Rutherford BR, Wall MM, Brown PJ et al. Patient expectancy as a mediator of placebo effects in antidepressant clinical trials. Am. J. Psychiatry 174(2), 135-142 (2017).

15 Zilcha-Mano S, Roose SP, Brown PJ, Rutherford BR. A machine learning approach to identifying placebo responders in late-life depression trials. Am. J. Geriatr. Psychiatry 26(6), 669-677 (2018).

16 Elsenbruch S, Enck P. Placebo effects and their determinants in gastrointestinal disorders. Nat. Rev. Gastroenterol. Hepatol. 12(8), 472-485 (2015).

17 Hall KT, Loscalzo J, Kaptchuk TJ. Genetics and the placebo effect: the placebome. Trends Mol. Med. 21(5), 285-294 (2015). 
18 Younger J, Gandhi V, Hubbard E, Mackey S. Development of the stanford expectations of treatment scale (SETS): a tool for measuring patient outcome expectancy in clinical trials. Clin. Trials 9(6), 767-776 (2012).

19 Mestre TA, Shah P, Marras C, Tomlinson G, Lang AE. Another face of placebo: the lessebo effect in Parkinson disease: meta-analyses. Neurology 82(16), 1402-1409 (2014).

20 Grahl A, Onat S, Büchel C. The periaqueductal gray and Bayesian integration in placebo analgesia. Elife 7, e32930 (2018). 